\title{
Weighted composition operators from Nevanlinna type spaces to weighted Bloch type spaces
}

\author{
Ajay K. Sharma, Ram Krishan and Elina Subhadarsini \\ School of Mathematics, Shri Mata Vaishno Devi University, Kakryal, Katra-182320, J\&K, India \\ E-mail: aksju_76@yahoo.com, ramk123.verma@gmail.com, elinamaths@gmail.com
}

\begin{abstract}
In this paper, we characterize metrically compact weighted composition operators from Nevanlinna type spaces to weighted Bloch type spaces.
\end{abstract}

2010 Mathematics Subject Classification. 47B33. 30H15, 30H30.

Keywords. Weighted composition operator, boundedness with respect to metric balls, metrical compactness, Nevanlinna type spaces, weighted Bloch type spaces.

\section{Introduction}

Let $\mathbb{D}$ be the open unit disk in the complex plane $\mathbb{C}, H(\mathbb{D})$ the space of all analytic functions on $\mathbb{D}$, $\mathbb{T}$ the boundary of $\mathbb{D}$ and $d \sigma$ denote the normalized Lebesgue measure on $\mathbb{T}$. The Nevanlinna class $N$ is defined as the set of all holomorphic functions $f$ on $\mathbb{D}$ such that

$$
\sup _{0 \leq r<1} \int_{\mathbb{T}} \log (1+|f(r \zeta)|) d \sigma(\zeta)<\infty
$$

It is well-known fact that every holomorphic function $f$ in the class $N$ has a finite non-tangential limit, denoted by $f^{*}$, almost everywhere on $\mathbb{T}$.

For each $1<p<\infty$, the Nevanlinna type space $N^{p}$ is a subspace of the Nevanlinna class $N$ defined as

$$
N^{p}=\left\{f \in H(\mathbb{D}):\|f\|_{N^{p}}=\sup _{0 \leq r<1}\left(\int_{\mathbb{T}}(\log (1+|f(r \zeta)|))^{p} d \sigma(\zeta)\right)^{1 / p}<\infty\right\} .
$$

$N^{p}$ is an $F$-space with respect to the translation-invariant metric $d_{N^{p}}(f, g)=\|f-g\|_{N^{p}}$. Moreover, the subharmonicity of $(\log (1+|f|))^{p}$ implies that $f \in N^{p}$ has the following growth estimation:

$$
\log (1+|f(z)|) \leq \frac{4^{1 / p}\|f\|_{N^{p}}}{\left(1-|z|^{2}\right)^{1 / p}}
$$

for $z \in \mathbb{D}$. Thus the convergence in $N^{p}$ gives the uniform convergence on compact subsets of $\mathbb{D}$. For more informations on Nevanlinna type spaces, see [1, 2, 3, 5, 6, 14].

Let $\nu$ be a continuous function (weight) on $\mathbb{D}$ such that $\nu(z)=\nu(|z|)$ for every $z \in \mathbb{D}$ and $\nu$ is normal, that is, there exist positive numbers $\eta$ and $\tau, 0<\eta<\tau$, and $\delta \in[0,1)$ such that

$$
\frac{\nu(r)}{(1-r)^{\eta}} \text { is decreasing on }[\delta, 1) \text { and } \lim _{r \rightarrow 1} \frac{\nu(r)}{(1-r)^{\eta}}=0 \text {; }
$$




$$
\frac{\nu(r)}{(1-r)^{\tau}} \text { is increasing on }[\delta, 1) \text { and } \lim _{r \rightarrow 1} \frac{\nu(r)}{(1-r)^{\tau}}=\infty .
$$

For such a weight $\nu$, the weighted Bloch-type space $\mathcal{B}_{\nu}$ on $\mathbb{D}$ is the space of all holomorphic functions $f$ on $\mathbb{D}$ such that

$$
\sup _{z \in \mathbb{D}} \nu(z)\left|f^{\prime}(z)\right|<\infty
$$

The little weighted Bloch-type space $\mathcal{B}_{\nu, 0}$ consists of all $f \in \mathcal{B}_{\nu}$ such that

$$
\lim _{|z| \rightarrow 1} \nu(z)\left|f^{\prime}(z)\right|=0
$$

Both spaces $\mathcal{B}_{\nu}$ and $\mathcal{B}_{\nu, 0}$ are Banach spaces with the norm

$$
\|f\|_{\mathcal{B}_{\nu}}=|f(0)|+\sup _{z \in \mathbb{D}} \nu(z)\left|f^{\prime}(z)\right|
$$

and $\mathcal{B}_{\nu, 0}$ is a closed subspace of $\mathcal{B}_{\nu}$. The compactness of a closed subset $L \subset \mathcal{B}_{\nu, 0}$ can be characterized as follows.

Lemma 1.1. A closed set $L$ in $\mathcal{B}_{\nu, 0}$ is compact if and only if it is bounded with respect to the norm $\|\cdot\|_{\mathcal{B}_{\nu}}$ and satisfies

$$
\lim _{|z| \rightarrow 1} \sup _{f \in L} \nu(z)\left|f^{\prime}(z)\right|=0 .
$$

This result for the case $\nu(z)=\left(1-|z|^{2}\right)$ was proved by Madigan and Matheson [7]. By a modification of their proof, we can prove the above lemma. Lemma 1.1 is a very useful tool in the study of the compactness of linear operators, when the range space is $\mathcal{B}_{\nu, 0}$ (see $[7,8,9,10]$, etc).

Let $\psi \in H(\mathbb{D})$ and $\varphi$ a holomorphic self-map of $\mathbb{D}$, the weighted composition operator $W_{\psi, \varphi}$ is a linear operator on $H(\mathbb{D})$ defined by $W_{\psi, \varphi} f=\psi \cdot f \circ \varphi$ for $f \in H(\mathbb{D})$. It is of interest to provide function-theoretic characterizations involving $\psi$ and $\varphi$ of boundedness and compactness of $W_{\psi, \varphi}$ acting between different function spaces. Given two linear topological vector spaces $X$ and $Y$, a linear operator $T: X \rightarrow Y$ is metrically bounded if there exists a constant $C>0$ such that $d_{Y}(T f, 0) \leq C d_{X}(f, 0)$ for $f \in X$. In general, the boundedness of $T$ and the metrical boundedness of $T$ do not coincide. However, if $X$ and $Y$ are Banach spaces, then the metrical boundedness of $T$ coincides with the boundedness of $T$. For more informations on the metrical boundedness, we can refer to papers [2,3]. Also recall that a linear operator $T: X \rightarrow Y$ is bounded with respect to metric balls if it takes every metric ball in $X$ into a metric ball in $Y$. Since a metric ball is also a bounded set, so boundedness with respect to metric balls also coincides with metrical boundedness, if $X$ and $Y$ are Banach spaces. For the compactness of linear operators, for example, composition operators or weighted composition operators on these spaces, we use the metrical compactness. Namely, $T: X \rightarrow Y$ is metrically compact if it takes every metric ball in $X$ into a relatively compact subset in $Y$. These operators on Nevanlinna type spaces have been studied by several authors (see $[1,2,3,8,9,10,11,12,13]$ ). Motivated by their work, in this paper, we will give characterizations for the boundedness with metric balls and the metrical compactness of $W_{\psi, \varphi}$ acting from Nevanlinna type spaces into weighted Bloch-type spaces. 


\section{Boundedness and compactness}

We first give sufficient conditions for $W_{\psi, \varphi}$ to be bounded with respect to metric balls.

Proposition 2.1. If $\psi \in H(\mathbb{D})$ and $\varphi$ a holomorphic self-map of $\mathbb{D}$ such that

$$
\sup _{z \in \mathbb{D}} \frac{\nu(z)\left|\psi(z) \varphi^{\prime}(z)\right|}{1-|\varphi(z)|^{2}} \exp \left\{\frac{c}{\left(1-|\varphi(z)|^{2}\right)^{1 / p}}\right\}<\infty
$$

and

$$
\sup _{z \in \mathbb{D}} \nu(z)\left|\psi^{\prime}(z)\right| \exp \left\{\frac{c}{\left(1-|\varphi(z)|^{2}\right)^{1 / p}}\right\}<\infty
$$

for every $c>0$, then $W_{\psi, \varphi}: N^{p} \rightarrow \mathcal{B}_{\nu}$ is bounded with respect to metric balls.

Proof. For $z \in \mathbb{D}$ and $\zeta \in \mathbb{T}$, we have

$$
1-|z+(1-|z|) \zeta / 2|^{2} \geq 1-(1+|z|)^{2} / 4 \geq\left(1-|z|^{2}\right) / 4 .
$$

Thus by Cauchy's integral formula and (1.1), we obtain that

$$
\left(1-|z|^{2}\right)\left|f^{\prime}(z)\right| \leq \frac{2}{\pi} \int_{\mathbb{T}}|f(z+(1-|z|) \zeta / 2)||d \zeta| \leq 4 \exp \left\{\frac{4^{1+1 / p}\|f\|_{N^{p}}}{\left(1-|z|^{2}\right)^{1 / p}}\right\}
$$

for each $z \in \mathbb{D}$. Hence we have that

$$
\begin{aligned}
\left\|W_{\psi, \varphi} f\right\|_{\mathcal{B}_{\nu}}= & |\psi(0) f(\varphi(0))|+\sup _{z \in \mathbb{D}} \nu(z)\left|\psi^{\prime}(z) f(\varphi(z))+\psi(z) \varphi^{\prime}(z) f^{\prime}(\varphi(z))\right| \\
\leq & |\psi(0)| \exp \left\{\frac{4^{1 / p}\|f\|_{N^{p}}}{\left(1-|\varphi(0)|^{2}\right)^{1 / p}}\right\}+\sup _{z \in \mathbb{D}} \nu(z)\left|\psi^{\prime}(z)\right| \exp \left\{\frac{4^{1 / p}|| f \|_{N^{p}}}{\left(1-|\varphi(z)|^{2}\right)^{1 / p}}\right\} \\
& +4 \sup _{z \in \mathbb{D}} \frac{\nu(z)\left|\psi(z) \varphi^{\prime}(z)\right|}{1-|\varphi(z)|^{2}} \exp \left\{\frac{\left.4^{1+1 / p}|| f\right|_{N^{p}}}{\left(1-|\varphi(z)|^{2}\right)^{1 / p}}\right\} .
\end{aligned}
$$

Combining the above inequality and condition (2.1) and condition (2.2), we see that $W_{\psi, \varphi}$ takes every metric ball in $N^{p}$ into a metric ball in $\mathcal{B}_{\nu}$, namely $W_{\psi, \varphi}: N^{p} \rightarrow \mathcal{B}_{\nu}$ is bounded with respect to metric balls.

Q.E.D.

Proposition 2.2. If $\psi \in H(\mathbb{D})$ and $\varphi$ a holomorphic self-map of $\mathbb{D}$ satisfying the conditions $(2.1)$ and (2.2) for every $c>0$, then $W_{\psi, \varphi}: N^{p} \rightarrow \mathcal{B}_{\nu}$ is bounded.

Proof. Suppose that conditions (2.1) and (2.2) hold, then proceeding as in the proof of Proposition 2.1 , we see that $W_{\psi, \varphi}\left(N^{p}\right) \subset \mathcal{B}_{\nu}$. Since $N^{p}$ is an $F$-space, hence by the closed graph theorem, we have that $W_{\psi, \varphi}: N^{p} \rightarrow \mathcal{B}_{\nu}$ is also bounded.

Q.E.D.

The main result of this paper, characterizes the metrical compactness of $W_{\psi, \varphi}: N^{p} \rightarrow \mathcal{B}_{\nu}$. In fact, we prove that boundedness of $W_{\psi, \varphi}: N^{p} \rightarrow \mathcal{B}_{\nu}$ with respect to metric balls is equivalent to metrically compactness of $W_{\psi, \varphi}: N^{p} \rightarrow \mathcal{B}_{\nu}$.

To prove the main result, we need the following lemma. 
Lemma 2.3. Suppose that $\psi \in H(\mathbb{D})$ and $\varphi$ a holomorphic self-map of $\mathbb{D}$ such that $W_{\psi, \varphi}\left(N^{p}\right) \subset$ $\mathcal{B}_{\nu}$. Then $W_{\psi, \varphi}: N^{p} \rightarrow \mathcal{B}_{\nu}$ is metrically compact if and only if for any sequence $\left\{f_{j}\right\}$ in $N^{p}$ with $\sup _{j}\left\|f_{j}\right\|_{N^{p}} \leq K$ and which converges to zero uniformly on compact subsets of $\mathbb{D},\left\{W_{\psi, \varphi} f_{j}\right\}$ converges to zero in $\mathcal{B}_{\nu}$.

Proof. This is an extension of a well-known result on the compactness of weighted composition operators on holomorphic function spaces. By (1.1), we see that any metrical bounded sequence in $N^{p}$ form a normal family. Hence an argument by using the Montel theorem proves this lemma proceeding on the same lines as the proof of Proposition 3.11 in [4].

Q.E.D.

Theorem 2.4. Let $\psi \in H(\mathbb{D})$ and $\varphi$ be a holomorphic self-map of $\mathbb{D}$. Then the following conditions are equivalent;

(i) $W_{\psi, \varphi}: N^{p} \rightarrow \mathcal{B}_{\nu}$ is bounded with respect to metric balls,

(ii) $W_{\psi, \varphi}: N^{p} \rightarrow \mathcal{B}_{\nu}$ is metrically compact,

(iii) $\psi \in \mathcal{B}_{\nu}$,

$$
\begin{gathered}
\sup _{z \in \mathbb{D}} \nu(z)\left|\psi(z) \varphi^{\prime}(z)\right|<\infty, \\
\lim _{|\varphi(z)| \rightarrow 1} \nu(z)\left|\psi^{\prime}(z)\right| \exp \left\{\frac{c}{\left(1-|\varphi(z)|^{2}\right)^{1 / p}}\right\}=0
\end{gathered}
$$

and

$$
\lim _{|\varphi(z)| \rightarrow 1} \frac{\nu(z)\left|\psi(z) \varphi^{\prime}(z)\right|}{1-|\varphi(z)|^{2}} \exp \left\{\frac{c}{\left(1-|\varphi(z)|^{2}\right)^{1 / p}}\right\}=0
$$

for any $c>0$.

Proof. Since $\mathcal{B}_{\nu}$ is a Banach space, so each bounded set is also in a metric ball in $\mathcal{B}_{\nu}$. Hence the metrical compactness of $W_{\psi, \varphi}$ from $N^{p}$ to $\mathcal{B}_{\nu}$ gives the boundedness with respect to metric balls. So implication (ii) $\Rightarrow$ (i) holds.

Now we will prove (iii) $\Rightarrow$ (ii). Assume that $\psi \in \mathcal{B}_{\nu}$ and that the conditions (2.4), (2.5) and (2.6) hold for every $c>0$. Let $\varepsilon>0$ be arbitrary. Then we can choose $r_{0} \in(0,1)$ such that

$$
\sup _{|\varphi(z)|>r_{0}} \nu(z)\left|\psi^{\prime}(z)\right| \exp \left\{\frac{c}{\left(1-|\varphi(z)|^{2}\right)^{1 / p}}\right\}<\varepsilon
$$

and

$$
\sup _{|\varphi(z)|>r_{0}} \frac{\nu(z)\left|\psi(z) \varphi^{\prime}(z)\right|}{1-|\varphi(z)|^{2}} \exp \left\{\frac{c}{\left(1-|\varphi(z)|^{2}\right)^{1 / p}}\right\}<\varepsilon
$$

for any $c>0$. Choose a sequence $\left\{f_{j}\right\}$ in $N^{p}$ such that $\sup _{j}\left\|f_{j}\right\|_{N^{p}} \leq K$ and $\left\{f_{j}\right\}$ converges to zero uniformly on compact subsets of $\mathbb{D}$. Since $\psi \in \mathcal{B}_{\nu}$ and (2.5) imply (2.2), and (2.4) and (2.6) imply (2.1), so by Proposition 2.2, we see that $W_{\psi, \varphi}\left(N^{p}\right) \subset \mathcal{B}_{\nu}$. The assumptions $\psi \in \mathcal{B}_{\nu}$ and (2.4) also imply that

$$
\sup _{|\varphi(z)| \leq r_{0}} \nu(z)\left|\psi^{\prime}(z)\right|\left|f_{j}(\varphi(z))\right| \leq \sup _{z \in \mathbb{D}} \nu(z)\left|\psi^{\prime}(z)\right| \cdot \max _{|w| \leq r_{0}}\left|f_{j}(w)\right| \rightarrow 0
$$


and

$$
\sup _{|\varphi(z)| \leq r_{0}} \nu(z)\left|\psi(z) \varphi^{\prime}(z)\right|\left|f_{j}^{\prime}(\varphi(z))\right| \leq \sup _{z \in \mathbb{D}} \nu(z)\left|\psi(z) \varphi^{\prime}(z)\right| \cdot \max _{|w| \leq r_{0}}\left|f_{j}^{\prime}(w)\right| \rightarrow 0
$$

as $j \rightarrow \infty$. Therefore, we have

$$
\sup _{|\varphi(z)| \leq r_{0}} \nu(z)\left|\left(W_{\psi, \varphi} f_{j}\right)^{\prime}(z)\right| \rightarrow 0
$$

as $j \rightarrow \infty$. On the other hand, it follows from (1.1) and (2.3) that

$$
\begin{aligned}
\sup _{|\varphi(z)|>r_{0}} \nu(z)\left|\left(W_{\psi, \varphi} f\right)^{\prime}(z)\right| \leq & \sup _{|\varphi(z)|>r_{0}} \nu(z)\left|\psi^{\prime}(z)\right| \exp \left\{\frac{4^{1 / p} K}{\left(1-|\varphi(z)|^{2}\right)^{1 / p}}\right\} \\
& +4 \sup _{|\varphi(z)|>r_{0}} \frac{\nu(z)\left|\psi(z) \varphi^{\prime}(z)\right|}{1-|\varphi(z)|^{2}} \exp \left\{\frac{4^{1+1 / p} K}{\left(1-|\varphi(z)|^{2}\right)^{1 / p}}\right\}<\varepsilon
\end{aligned}
$$

Thus we see that

$$
\limsup _{j \rightarrow \infty}\left\|W_{\psi, \varphi} f_{j}\right\|_{\mathcal{B}_{\nu}}<\varepsilon
$$

Since $\varepsilon>0$ is arbitrary, we have that $\left\|W_{\psi, \varphi} f_{j}\right\|_{\mathcal{B}_{\nu}} \rightarrow 0$ as $j \rightarrow \infty$. Hence Lemma 2.3 shows that $W_{\psi, \varphi}: N^{p} \rightarrow \mathcal{B}_{\nu}$ is metrically compact.

Finally we prove the implication (i) $\Rightarrow$ (iii). By taking the constant function $f(z)=1$ in $N^{p}$, we have that $\psi \in \mathcal{B}_{\nu}$. Again by taking $f(z)=z$ in $N^{p}$ and using facts that $|\varphi(z)|<1$ and $\psi \in \mathcal{B}_{\nu}$, we have that

$$
\sup _{z \in \mathbb{D}} \nu(z)\left|\psi(z) \varphi^{\prime}(z)\right|<\infty .
$$

Fix $c>0$ and put $w=\varphi(z)$. We define the following function

$$
f_{w}(v)=\left\{3 \frac{1-|w|^{2}}{(1-\bar{w} v)^{2}}-2 \frac{\left(1-|w|^{2}\right)^{2}}{(1-\bar{w} v)^{3}}\right\} \exp \left\{c\left\{3 \frac{1-|w|^{2}}{(1-\bar{w} v)^{2}}-2 \frac{\left(1-|w|^{2}\right)^{2}}{(1-\bar{w} v)^{3}}\right\}^{1 / p}\right\}
$$

Then we can easily see that the family $\left\{f_{w}\right\}$ forms a metric ball in $N^{p}$. Also we have that

$$
\begin{aligned}
f_{w}^{\prime}(v)= & \left\{\frac{6 c \bar{w}}{p}\left\{3 \frac{1-|w|^{2}}{(1-\bar{w} v)^{2}}-2 \frac{\left(1-|w|^{2}\right)^{2}}{(1-\bar{w} v)^{3}}\right\}^{1 / p}+6 \bar{w}\right\}\left\{\frac{1-|w|^{2}}{(1-\bar{w} v)^{3}}-\frac{\left(1-|w|^{2}\right)^{2}}{(1-\bar{w} v)^{4}}\right\} \\
& \times \exp \left\{c\left\{3 \frac{1-|w|^{2}}{(1-\bar{w} v)^{2}}-2 \frac{\left(1-|w|^{2}\right)^{2}}{(1-\bar{w} v)^{3}}\right\}^{1 / p}\right\} .
\end{aligned}
$$

Since $\left\{W_{\psi, \varphi} f_{w}\right\}$ is a metric ball in $\mathcal{B}_{\nu}$, there is a positive constant $C$ which is independent of $w=\varphi(z)$ such that $\left\|W_{\psi, \varphi} f_{w}\right\|_{\mathcal{B}_{\nu}} \leq C$. Thus by using the fact that $f_{w}^{\prime}(w)=0$, we obtain that

$$
\begin{aligned}
C & \geq \nu(z)\left|\left(W_{\psi, \varphi} f_{w}\right)^{\prime}(z)\right| \\
& =\frac{\nu(z)\left|\psi^{\prime}(z)\right|}{1-|\varphi(z)|^{2}} \exp \left\{\frac{c}{\left(1-|\varphi(z)|^{2}\right)^{1 / p}}\right\},
\end{aligned}
$$


and so

$$
\nu(z)\left|\psi^{\prime}(z)\right| \exp \left\{\frac{c}{\left(1-|\varphi(z)|^{2}\right)^{1 / p}}\right\} \leq C\left(1-|\varphi(z)|^{2}\right) .
$$

Taking limit as $|\varphi(z)| \rightarrow 1$ on both sides of the above inequality, we get (2.5). Again fix $c>0$, put $w=\varphi(z)$ and consider the following function

$$
h_{w}(v)=\left\{\frac{1-|w|^{2}}{(1-\bar{w} v)^{2}}-\frac{\left(1-|w|^{2}\right)^{2}}{(1-\bar{w} v)^{3}}\right\} \exp \left\{c\left\{3 \frac{1-|w|^{2}}{(1-\bar{w} v)^{2}}-2 \frac{\left(1-|w|^{2}\right)^{2}}{(1-\bar{w} v)^{3}}\right\}^{1 / p}\right\} .
$$

Then once again we can easily see that $\left\{h_{w}\right\}$ forms a metric ball in $N^{p}$ and $h_{w}(w)=0$. Also we have that

$$
\begin{aligned}
h_{w}^{\prime}(v)= & {\left[\bar{w}\left\{2 \frac{1-|w|^{2}}{(1-\bar{w} v)^{3}}-3 \frac{\left(1-|w|^{2}\right)^{2}}{(1-\bar{w} v)^{4}}\right\}+\frac{6 c}{p} \bar{w}\left\{\frac{1-|w|^{2}}{(1-\bar{w} v)^{2}}-\frac{\left(1-|w|^{2}\right)^{2}}{(1-\bar{w} v)^{3}}\right\}\right.} \\
& \left.\times\left\{3 \frac{\left(1-|w|^{2}\right)}{(1-\bar{w} v)^{2}}-2 \frac{\left(1-|w|^{2}\right)^{2}}{(1-\bar{w} v)^{3}}\right\}^{-1+1 / p}\left\{\frac{1-|w|^{2}}{(1-\bar{w} v)^{3}}-\frac{\left(1-|w|^{2}\right)^{2}}{(1-\bar{w} v)^{4}}\right\}\right] \\
\times & \exp \left\{c\left\{3 \frac{1-|w|^{2}}{(1-\bar{w} v)^{2}}-2 \frac{\left(1-|w|^{2}\right)^{2}}{(1-\bar{w} v)^{3}}\right\}^{1 / p}\right\} .
\end{aligned}
$$

Therefore, we have that

$$
h_{w}^{\prime}(w)=\frac{-\bar{w}}{\left(1-|w|^{2}\right)^{2}} \exp \left\{\frac{c}{\left(1-|\varphi(z)|^{2}\right)^{1 / p}}\right\} .
$$

Since $\left\{W_{\psi, \varphi} h_{w}\right\}$ is a metric ball in $\mathcal{B}_{\nu}$, there is a positive constant $C$ which is independent of $w=\varphi(z)$ such that $\left\|W_{\psi, \varphi} h_{w}\right\|_{\mathcal{B}_{\nu}} \leq C$. Thus we obtain that

$$
\begin{aligned}
C & \geq \nu(z)\left|\left(W_{\psi, \varphi} f_{w}\right)^{\prime}(z)\right| \\
& =\frac{\nu(z)\left|\psi(z) \varphi^{\prime}(z)\right||\varphi(z)|}{\left(1-|\varphi(z)|^{2}\right)^{2}} \exp \left\{\frac{c}{\left(1-|\varphi(z)|^{2}\right)^{1 / p}}\right\},
\end{aligned}
$$

and so

$$
\frac{\nu(z)\left|\psi(z) \varphi^{\prime}(z)\right|}{1-|\varphi(z)|^{2}} \exp \left\{\frac{c}{\left(1-|\varphi(z)|^{2}\right)^{1 / p}}\right\} \leq \frac{C p\left(1-|\varphi(z)|^{2}\right)}{|\varphi(z)|} .
$$

Taking limit as $|\varphi(z)| \rightarrow 1$ on both sides of the above inequality, we get (2.6). This completes the proof.

Q.E.D.

Next we will investigate the compactness of operators $W_{\psi, \varphi}: N^{p} \rightarrow \mathcal{B}_{\nu, 0}$.

Proposition 2.5. If $\psi \in H(\mathbb{D})$ and $\varphi$ a holomorphic self-map of $\mathbb{D}$ such that

$$
\begin{gathered}
\lim _{|z| \rightarrow 1} \nu(z)\left|\psi^{\prime}(z)\right| \exp \left\{\frac{c}{\left(1-|\varphi(z)|^{2}\right)^{1 / p}}\right\}=0 \\
\lim _{|z| \rightarrow 1} \frac{\nu(z)\left|\psi(z) \varphi^{\prime}(z)\right|}{1-|\varphi(z)|^{2}} \exp \left\{\frac{c}{\left(1-|\varphi(z)|^{2}\right)^{1 / p}}\right\}=0
\end{gathered}
$$

for all $c>0$, then $W_{\psi, \varphi}: N^{p} \rightarrow \mathcal{B}_{\nu, 0}$ is bounded with respect to metric balls. Also we obtain that $W_{\psi, \varphi}\left(N^{p}\right) \subset \mathcal{B}_{\nu, 0}$. 
Proof. Since $W_{\psi, \varphi}: N^{p} \rightarrow \mathcal{B}_{\nu}$ is bounded with respect to metric balls by Proposition 2.1, we only need to prove that $W_{\psi, \varphi}(L) \subset \mathcal{B}_{\nu, 0}$ for any metric balls $L$ in $N^{p}$. However, the inequalities (1.1) and (2.3) show that

$$
\begin{aligned}
\nu(z)\left|\left(W_{\psi, \varphi} f\right)^{\prime}(z)\right| & \leq \nu(z)\left|\varphi^{\prime}(z)\right| \exp \left\{\frac{4^{1 / p}\|f\|_{N^{p}}}{\left(1-|\varphi(z)|^{2}\right)^{1 / p}}\right\} \\
& +4 \frac{\nu(z)\left|\varphi^{\prime}(z)\right|}{1-|\varphi(z)|^{2}} \exp \left\{\frac{4^{1+1 / p}\|f\|_{N^{+}}}{\left(1-|\varphi(z)|^{2}\right)^{1 / p}}\right\}
\end{aligned}
$$

which holds for each $f \in L$. Thus the conditions (2.7) and (2.8) imply that $\nu(z)\left|\left(W_{\psi, \varphi} f\right)^{\prime}(z)\right| \rightarrow 0$ as $|z| \rightarrow 1$. This completes the proof.

Q.E.D.

Theorem 2.6. Let $\psi \in H(\mathbb{D})$ and $\varphi$ be a holomorphic self-map of $\mathbb{D}$. Then the following conditions are equivalent;

(i) $W_{\psi, \varphi}: N^{p} \rightarrow \mathcal{B}_{\nu, 0}$ is bounded with respect to metric balls,

(ii) $W_{\psi, \varphi}: N^{p} \rightarrow \mathcal{B}_{\nu}$ is bounded with respect to metric balls, $\psi \in \mathcal{B}_{\nu, 0}$ and $\lim _{|z| \rightarrow 1} \nu(z)\left|\psi(z) \varphi^{\prime}(z)\right|=0$,

(iii) $W_{\psi, \varphi}: N^{p} \rightarrow \mathcal{B}_{\nu, 0}$ is metrically compact,

(vii) $\psi$ and $\varphi$ satisfy the conditions (2.7) and (2.8).

Proof. The implication (iii) $\Rightarrow$ (i) is obvious. Also we can easily see that (i) $\Rightarrow$ (ii) are holds. In fact, we may consider the function $f(z)=1$ in $N^{p}$. Then $\|f\|_{N^{p}}=\log 2$, and so $f$ is in some metric balls in $N^{p}$. This shows that $\psi \in \mathcal{B}_{\nu, 0}$. Again, consider the function $f(z)=z$ in $N^{p}$. Once again $\|f\|_{N^{p}} \leq \log 2$, and so $f$ is in some metric balls in $N^{p}$. Thus using $\psi \in \mathcal{B}_{\nu, 0}$, we can shows that $\lim _{|z| \rightarrow 1} \nu(z)\left|\psi(z) \varphi^{\prime}(z)\right|=0$.

To prove (ii) $\Rightarrow$ (iv), we take a sequence $\left\{z_{j}\right\}$ in $\mathbb{D}$ with $\left|z_{j}\right| \rightarrow 1$ as $j \rightarrow \infty$. Then

$$
\begin{aligned}
& \limsup _{|z| \rightarrow 1} \frac{\nu(z)\left|\psi(z) \varphi^{\prime}(z)\right|}{1-|\varphi(z)|^{2}} \exp \left\{\frac{c}{\left(1-|\varphi(z)|^{2}\right)^{1 / p}}\right\} \\
& =\lim _{j \rightarrow \infty} \frac{\nu\left(z_{j}\right)\left|\psi\left(z_{j}\right) \varphi^{\prime}\left(z_{j}\right)\right|}{1-\left|\varphi\left(z_{j}\right)\right|^{2}} \exp \left\{\frac{c}{\left(1-\left|\varphi\left(z_{j}\right)\right|^{2}\right)^{1 / p}}\right\} .
\end{aligned}
$$

If $\sup _{j \geq 1}\left|\varphi\left(z_{j}\right)\right|<1$, then the assumption $\psi \in \mathcal{B}_{\nu, 0}$ implies that the right limit in the equation (2.9) is equal to 0 , and so we obtain the condition (2.7). If $\sup _{j \geq 1}\left|\varphi\left(z_{j}\right)\right|=1$, then we can choose a subsequence $\left\{z_{j_{k}}\right\} \subset\left\{z_{j}\right\}$ such that $\left|\varphi\left(z_{j_{k}}\right)\right| \rightarrow 1$ as $k \rightarrow \infty$. Since $W_{\psi, \varphi}: N^{p} \rightarrow \mathcal{B}_{\nu}$ is bounded with respect to metric balls, by Theorem $2.4, \psi$ and $\varphi$ satisfy

$$
\lim _{|\varphi(z)| \rightarrow 1} \frac{\nu(z)\left|\psi(z) \varphi^{\prime}(z)\right|}{1-|\varphi(z)|^{2}} \exp \left\{\frac{c}{\left(1-|\varphi(z)|^{2}\right)^{1 / p}}\right\}=0
$$


for any $c>0$. By (2.9) and (2.10) we have that

$$
\begin{aligned}
& \limsup _{|z| \rightarrow 1} \frac{\nu(z)\left|\psi(z) \varphi^{\prime}(z)\right|}{1-|\varphi(z)|^{2}} \exp \left\{\frac{c}{\left(1-|\varphi(z)|^{2}\right)^{1 / p}}\right\} \\
& =\lim _{k \rightarrow \infty} \frac{\nu\left(z_{j_{k}}\right)\left|\psi\left(z_{j}\right) \varphi^{\prime}\left(z_{j_{k}}\right)\right|}{1-\left|\varphi\left(z_{j_{k}}\right)\right|^{2}} \exp \left\{\frac{c}{\left(1-\left|\varphi\left(z_{j_{k}}\right)\right|^{2}\right)^{1 / p}}\right\} \\
& \leq \limsup _{|\varphi(z)| \rightarrow 1} \frac{\nu(z)\left|\psi(z) \varphi^{\prime}(z)\right|}{1-|\varphi(z)|^{2}} \exp \left\{\frac{c}{\left(1-|\varphi(z)|^{2}\right)^{1 / p}}\right\}=0 .
\end{aligned}
$$

This implies that (2.7) holds. Similarly, we can show that (2.8) also holds.

Finally we will prove the implication (iv) $\Rightarrow$ (iii). Take any metric ball $L_{N^{p}}$ in $N^{p}$. Then there is a constant $K>0$ such that $\|f\|_{N^{p}} \leq K$ for any $f \in L_{N^{p}}$. For any $f \in L_{N^{p}}$ and $z \in \mathbb{D}$ we have that

$$
\begin{aligned}
\nu(z)\left|\left(W_{\psi, \varphi} f\right)^{\prime}(z)\right| & \leq \nu(z)\left|\psi^{\prime}(z)\right| \exp \left\{\frac{4^{1 / p} K}{\left(1-|\varphi(z)|^{2}\right)^{1 / p}}\right\} \\
& +4 \frac{\nu(z)\left|\psi(z) \varphi^{\prime}(z)\right|}{1-|\varphi(z)|^{2}} \exp \left\{\frac{4^{1+1 / p} K}{1-|\varphi(z)|^{2}}\right\} .
\end{aligned}
$$

Combining this with (2.7) and (2.8), we obtain

$$
\lim _{|z| \rightarrow 1} \sup _{f \in L_{N} p} \nu(z)\left|\left(W_{\psi, \varphi} f\right)^{\prime}(z)\right|=0,
$$

and so Lemma 1.1 shows that $W_{\psi, \varphi}\left(L_{N^{p}}\right)$ is compact in $\mathcal{B}_{\nu, 0}$ for any metric ball $L_{N^{p}}$. This means that $W_{\psi, \varphi}: N^{p} \rightarrow \mathcal{B}_{\nu, 0}$ is metrically compact. The proof is accomplished.

Q.E.D.

Acknowledgments. The second author is thankful to DST (India) for Inspire fellowship: DST/Inspire fellowship/2013/281.

\section{References}

[1] J.S. Choa and H.O. Kim, Composition operators between the Nevanlinna-type spaces, J. Math. Anal. Appl. 257 (2001), 378-402.

[2] J.S. Choa, H.O. Kim and J.H. Shapiro, Compact composition operators on the Smirnov class, Proc. Amer. Math. Soc. 128 (2000), 2297-2308.

[3] B.R. Choe, H. Koo and W. Smith, Carleson measures for the area Nevanlinna spaces and applications, J. Anal. Math. 104 (2008), 207-233.

[4] C.C. Cowen and B. D. MacCluer, Composition operators on spaces of analytic functions, CRC press, Boca Raton, New York, 1995.

[5] J.B. Garnett, Bounded Analytic Functions, Academic Press, New York, 1981.

[6] H.O. Kim, On an F-algebra of holomorphic functions, Can. J. Math. 40 (1988), 718-741. 
[7] K. Madigan and A. Matheson, Compact composition operators on the Bloch space, Trans. Amer. Math. Soc. 347 (1995), 2679-2687.

[8] A.K. Sharma, Products of multiplication, composition and differentiation operators between weighted Bergman-Nevanlinna and Bloch-type spaces, Turkish J. Math. 35 (2011), 275-291.

[9] A.K. Sharma, Weighted composition operators from Cauchy integral transforms to logarithmic weighted-type spaces. Ann. Funct. Anal. 4 (2013), 163-174.

[10] A.K. Sharma and S. Ueki, Composition operators from Nevanlinna type spaces to Bloch type spaces, Banach J. Math. Anal., 6 (2012), 112-123.

[11] S. Stević, Weighted differentiation composition operators from $H^{\infty}$ and Bloch spaces to nth weighted-type spaces on the unit disk, Appl. Math. Comput. 216 (2010), 3634-3641.

[12] S. Stević, Weighted composition operators from Bergman-Privalov-type spaces to weighted-type spaces on the unit ball, Appl. Math. Comput. 217 (2010), 1939-1943.

[13] S. Stević and A.K. Sharma, Composition operators from Bergman Privalov spaces to Zygmund spaces, Ann. Polon. Math. 105 (2012), 77-86.

[14] N. Yanagihara, Bounded subsets of some spaces of holomorphic functions, Sci. Papers College Gen. Ed. Univ. Tokyo 23 (1973), 19-28. 